\title{
Estudio comparativo de dos tipos de agujas en hemodiafiltración de alta eficacia
}

\author{
Ana Vanessa Fernández Martínez, Verena Romero Willhoft, Laura Pérez Valencia, Alicia Moreno Vallejo, \\ Tania Ortega Gómez, Salvadora Soto Ureña
}

\section{Fresenius Medical Care Services Cartagena y San Pedro del Pinatar. Cartagena. Murcia}

\section{Introducción:}

La hemodiafiltración on line post dilucional (HDFOL) es la más eficaz en depuración de moléculas de diferentes pesos. El volumen convectivo (VTR) puede estar relacionado con la supervivencia del paciente. Asi, en el ESHOL, se reduce la mortalidad un $33 \%$ con VTR medio de 20,8l. Los subanálisis del estudio CONTRAST y TURKEY encuentran esa reducción de mortalidad en los tertiles de mayor volumen de reinfusión. Por otro lado, la dosis de diálisis ( $\mathrm{Kt}$ ) es considerada fundamental en la diálisis adecuada y en la supervivencia del paciente. Ambos, Kt y VTR están influenciados por el flujo sanguíneo, habiendo sido debatido el uso de diferentes calibres de aguja en lo referente a resultados de eficiencia y valoración de dolor. El catéter fístula supercath, una nueva cánula de punción de fluoruroplástico, presenta una menor calibre de punción con un mayor calibre interno, permitiendo alcanzar mayores flujos sanguíneos con menor daño en la pared del acceso.

\section{Objetivo:}

Comparar la eficacia, seguridad, comodidad y sensación de dolor entre el catéter fístula y las agujas convencionales en el paciente en HDFOL de alta eficacia.

\section{Material y métodos:}

Estudio prospectivo cruzado sobre población prevalente en HDFOL posdilucional, con acceso vascular en territorio braquial o prótesis de PTFE. 33 pacientes, 17 durante 12 sesiones ( $F 1$ ) con supercath y el resto con aguja convencional 14 o $15 \mathrm{G}$. Se cruzan a partir de la 12 sesion (F2). Posteriormente 2 fases más de 12 sesiones $(F 1=F 3, F 2=F 4)$. Todas las sesiones con ST 5008 y la misma pauta de diálisis. Se analizan variables demográficas, hemodinámicas del acceso vascular, de seguridad y escala de dolor y comodidad pre-post para el enfermero. Analisis estadistico SPSS 13.0. Significación $p<0,05$.

\section{Resultados}

33 pacientes, 60,4\% hombres, 67,6 años de edad, 94\% fístulas nativas, $75 \% 15$ y $24,2 \% 14$ G. 1584 sesiones.

No diferencias en Presión arterial, Presión venosa y recirculación. Sí en flujo sanguíneo, supercath $463,95 \mathrm{ml} /$ min versus convencional $444,68 \mathrm{ml} / \mathrm{min}(p=0,02)$.

En eficacia, diferencias significativas en Kt $(p=0,04)$, $\operatorname{VTR}(p=0,00)$ y litros de sangre dializada $(p=0,01)$, supercath $(63,1),(27,4),(113,1)$ versus convencional $(60,9),(25,3),(108,5)$ respectivamente.

No diferencias en extravasaciones, aunque se repiten más punciones con supercath $(10,4 \%$ versus $3,5 \%, p<$ 0,001).

El $48,9 \%$ de las sesiones con supercath presentan tiempo de hemostasia entre 5 y 10 minutos, frente al $40,4 \%$ con convencional $(p=0,018)$. No hay diferencias en el estado final de cámara y dializador.

Valoración enfermera de comodidad significativamente $(p=0,00)$ mejor con las agujas convencionales tanto en conexión como desconexión. Percepción de dolor para el paciente es mayor con supercath. 


\section{Conclusiones:}

El uso del catéter fístula supercath nos permite conseguir mayor flujo sanguineo, sin incremento de presiones tanto arteriales como venosas ni recirculación, lo que nos hace conseguir mayor eficacia dialitica y VTR. Si bien, la valoración tanto del enfermero como del paciente en escala de seguridad, comodidad y dolor es peor con el supercath, estos resultados se producen fundamentalmente en las fases 1 y 2 , por lo que una vez más se requiere del conocimiento y perfeccionamiento de la técnica del enfermero nefrológico.

\section{Referencias Bibliográficas}

1. Maduell F, Moreso F, Pons M, Ramos R, MoraMacià J, Carreras J et al. High-Efficiency PostdiIution Online Hemodiafiltration. Reduces All-Cause Mortality in Hemodialysis Patients. Journal of the American Society of Nephrology. 2013; 24: 1-11.

2. Muriel P.C.Grooteman, Marius A, Michiel L Bots, E. Lars Penne, Neelke C. van der Weerd, Albert H.A. Mazairac et al. Effect of Online Hemodiafiltration on All-Cause Moortality and Cardiovascular Outcomes. 2012; 23: 1087-1096.

3. Ercan Ok, Gulay Asci, Ebru Sevinc Ok, Fatih KirceIli, Mumtaz Yilmaz, Ender Hur et al. Comparison of postdilution online hemodiafiltration ahn hemodialysis (Turkish HDF Study). En : Libro de comunicaciones; EDTA Praga 2011.

4. Martínez Ocaña 0, Rodríguez Estaire J, Ruiz Sanz B, Martínez Navarro JA, Merida Herrero E. Catéter-fístula: una nueva alternativa en la punción de accesos vasculares. Rev Soc Esp Enferm Nefrol. 2010; 13 (2): 105 -111.

5. Maduell F y Arias M. Indicaciones y prescripción de la hemodiafiltración. Dial Trasp 2008: 29(2): 62-66.

6. Maduell F, García H, Hernández-Jara J y cols. Comparación de la infusión predilucional versus postdilucional en la hemodiafiltración en línea. Nefrología 1998; 18; Supl 3-49.

7. Maduell F. Convección versus difusión. ¿Ha llegado el momento del cambio? Nefrología 2009; 29(6):589-593.

8. Alfaro A, Beltrán MI, Gallego B y cols. HDF en línea en nuestros pacientes: calidad de vida y capa- cidad funcional. Rev Soc Esp Enferm Nefrol 2006; 9 (3): 158-163.

9. Canaud B, Braga-Gresham JL, Marshal MR y cols. Mortality risk for patients receiving haemodiafiltration versus haemodialysis: European results from the DOPPS. Kidney Int. 2006; 69: 2087-2093.

10. Jirka T, Cesare S, Di Benedetto y cols. Mortality risk for patients receiving hemodiafiltration versus hemodialysis. Kidney Int 2006, 70, 1524.

11. Fernández AV, Soto $S$, Arenas M y cols. Comparación de infusión automática respecto a manual en hemodiafiltración on line postdilucional. $17 \mathrm{Rev}$ Soc Esp Enferm Nefrol 2010; 13 (1):17-22.

12. Held PJ, Port FK, Wolfe RA y cols: The dose of hemodialysis and patients mortality. Kidney Int 50: 550-556, 1996.

13. Fernández $\mathrm{AV}$, Soto $\mathrm{S}$, Arenas $\mathrm{M}$ y cols Estudio comparativo de la dosis de dialisis medida por (Kt) y KtV. 21 Rev Soc Esp Enferm Nefrol 2009; 12(2): 97-102.

14. Jarriz A, Bardale E, Pulido A y cols. Efecto del calibre de la aguja sobre la eficiencia de la hemodiálisis. Rev Soc Esp Enferm Nefrol 2006; 9 (2): 128-131.

15. Crespo $R$, Casas $R$, Muñoz $J$ y cols. Influencia del calibre de la aguja sobre el grado de dolor originado en la punción de la fístula arteriovenosa.

16. Pérez B, Teruel JL, Cerro P y cols. Influencia del calibre de las agujas en la eficacia de la diálisis. Rev Soc Esp Enferm Nefrol 2001; 4 (3): 86-87.

17. Cañada R, Puig J, Ferrero S. Grado de dolor al pinchar la fístula arteriovenosa con agujas congeladas comparando con agujas a temperatura ambiente. Rev Soc Esp Enferm Nefrol 2004; 7 (2): 74-76.

18. Sanchez Villar, I y cols. Calibre de la aguja y calidad de la hemodiálisis. Comunicación en el XXIV Congreso de la SEDEN. Valencia. 1999; 120-124.

19. Fernández AV, Caparrós V, Horrillo F, Pérez L, Fernández-Caro J, Pereira MS et al. La eficacia de la hemodiafiltración es dependiente del flujo sanguineo. Entonces, ¿Por qué no incrementamos el calibre de las agujas?. En: Libro de comunicaciones del XXXVI Congreso e la Sociedad Española de Enfermería Nefrologia; Sevilla 2011. 